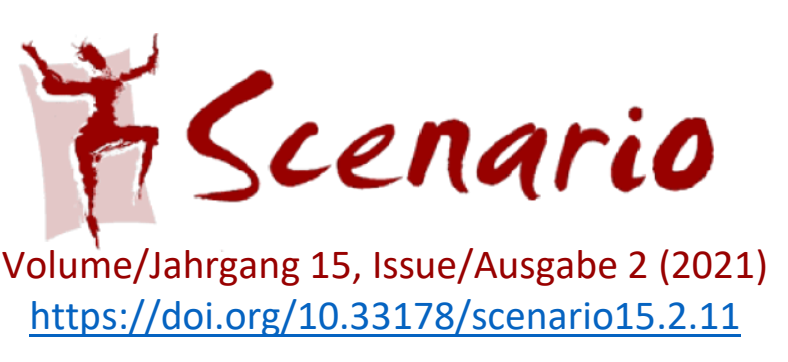

\title{
Rezension: Schappert, P. (2020) Mit Dramagrammatik zu grammatikalischer Kompetenz. Eine empirische Untersuchung im Rahmen von Alphabetisierungskursen für Erwachsene. Tectum Verlag
}

Susanne Even

Die in Buchrezensionen vertretenen Ansichten und Meinungen sind die der jeweiligen Rezensent:innen und reflektieren nicht notwendigerweise die Position von SCENARIO.

Achtzehn Jahre nach der Veröffentlichung von Drama Grammatik untersucht Petra Schappert in ihrer Dissertation an der Phillips-Universität Marburg die Effizienz des dramagrammatischen Ansatzes im Kontext von Alphabetisierungskursen für Erwachsene. Dieses Projekt ist intuitiv bestechend, führt Dramagrammatik zunächst doch spielerischinduktiv an fremdsprachliche Grammatikphänomene heran und könnte damit eine echte Lernalternative für erwachsene Analphabeten darstellen.

Die empirische Studie wurde 2017-2018 in einem Integrationskurs mit Alphabetisierung bei den Henke-Schulungen in Stuttgart durchgeführt, bei dessen Teilnehmenden es sich überwiegend um primäre Analphabet:innen unterschiedlicher Altersgruppen, Erstsprachen, Herkunftsländer und Aufenthaltsdauer in Deutschland handelte. Eine Testgruppe (9 Lernende) wurde dramagrammatisch unterrichtet, während eine Kontrollgruppe (7 Lernende) die gängige kommunikative Unterweisung bekam.

Angesichts der niedrigen Anzahl und hohen Bandbreite der Testpersonen drängt sich sofort das Prädikat mutig auf. Die Datenerhebungen fanden unter erschwerten Bedingungen statt: Sinn und Zweck der Studie musste von Dolmetscher:innen übermittelt werden, die Zahl der Teilnehmenden fluktuierte und die Leistungsmessungen gestalteten sich problematisch. Die Zusammenarbeit mit einer anderen Lehrkraft lief nicht immer rund, und die zur Verfügung stehenden Unterrichtsräume ließen schwer zu wünschen übrig.

Nichtsdestoweniger hat Schappert sich dieser Herausforderung gestellt. Kapitel 1 des Buches beschreibt Problemstellung, Ziel, Rahmenbedingungen und Aufbau der Arbeit, gefolgt von Kapitel 2, in dem es um Alphabetisierung und Grammatikvermittlung geht. Kapitel 3 
beschäftigt sich mit theoretischen Prinzipien und praktischen Umsetzungen von kommunikativem Grammatikunterricht. In Kapitel 4 geht es ausführlich um Dramapädagogik. Präsentiert wird ein Überblick über Drama in Education in Großbritannien (4.2) und Theaterpädagogik in Deutschland (4.3). Zentrale Forschungsarbeiten von Manfred Schewe, Elektra Tselikas, Ruth Huber und Benedikt Kessler werden referiert (4.4) und Chancen und Grenzen von Dramapädagogik besprochen (4.5). Im Anschluss daran stellt die Verfasserin unterschiedliche Formen des Aufbaus dramapädagogischer Unterrichtseinheiten (4.6 und 4.7) vor und diskutiert didaktische und logistische Anforderungen dramapädagogischen Lehrens und Lernens (4.8 und 4.9). Das Kapitel schließt mit einer Stellungnahme zu den Grenzen der Dramapädagogik in der praktischen Umsetzung (4.10) ab.

Kapitel 5 beginnt mit einer Zusammenfassung des dramagrammatischen Modells nach Even (2003) (5.1) sowie dessen Umsetzung in verschiedenen Theatercamps für Grundschulkinder und argumentiert, dass die Übertragbarkeit des Modells auf Alphabetisierungskurse zentrale Modifikationen erfordert (5.2-5.3). In Unterkapitel 5.4 legt die Verfasserin dar, dass Alphabetisierungskurse trotz ihrer Situierung im Zielland die Charakteristika von Fremdsprachenunterricht aufweisen, geht auf sensible Phasen beim L2-Erwerb ein, diskutiert explizites versus implizites Lernen sowie individuelle, das Lernen bestimmende Faktoren und fasst gängige Fremdsprachenlernhypothesen zusammen. Zum Schluss werden Gründe für die Wahl der Dramagrammatik als der empirischen Untersuchung zu Grunde liegenden Methodologie angeführt.

In Kapitel 6 werden Forschungsfragen formuliert und Hypothesen aufgestellt. Es soll herausgefunden werden, ob Grammatikunterricht im Alphabetisierungsprozess erfolgreich sein kann, inwieweit Dramagrammatik bzw. die Kommunikative Methode von den jeweiligen Versuchsgruppen angenommen wird und wie Effektivität und Nachhaltigkeit beider Methoden bewertet werden kann. Die Hypothesen postulieren einen positiven Zusammenhang zwischen der Umsetzung von Dramagrammatik und Lernerfolgen sowie positive Akzeptanz der Methode auf Seiten der Teilnehmenden.

Kapitel 7 umreißt die Rahmenbedingungen der Datenerhebung sowie Formen der Datenanalyse (schriftliche/mündliche Tests zu verschiedenen Zeitpunkten der Datenerhebung sowie Teilnehmer:inneninterviews). Weiterhin gibt es detaillierte Beschreibungen der Unterrichtsablaufpläne zu den vier Grammatikthemen Singular/Plural, Konjugation des Präsens, Wortstellung und Possessivartikel. In Kapitel 8 werden die empirischen Datensätze quantitativ und visuell gebündelt und in Kapitel 9 analysiert. Kapitel 10 schließt mit einer Zusammenfassung der Ergebnisse. Das sich daran anschließende Literaturverzeichnis und ein umfangreicher Anhang von 110 Seiten bilden das Ende des Bandes. 
Mit Dramagrammatik zu grammatikalischer Kompetenz betritt Schappert Neuland, daran besteht kein Zweifel. Es wäre allerdings wünschenswert gewesen, wenn die Verfasserin detailliertere inhaltliche und didaktische Anleitung bekommen hätte, denn die Arbeit weist deutliche Schwächen in Aufbau, Durchführung und Analyse auf.

Zunächst hätte sich eine ausgewogenere inhaltliche Progression positiv auf die Lesbarkeit ausgewirkt: Die ersten drei Kapitel sind mit insgesamt 17 Seiten erstaunlich knapp gehalten; insbesondere zum Thema Alphabetisierung und Grammatikunterricht (3 Seiten) hätte man sich eine eingehende Auseinandersetzung mit Alphabetisierungskonzepten der vergangenen Jahre gewünscht. Im Gegensatz dazu scheinen Kapitel 4 (70 Seiten) und Kapitel 5 (47 Seiten) überladen mit unterschiedlichen Themenkomplexen, deren inhaltliche Darstellung lückenhaft bleibt. So lässt Kapitel 4 beispielsweise zentrale Autor:innen wie Gavin Bolton (Drama in Education), Hans-Martin Ritter und Ulrike Hentschel (Theaterpädagogik) sowie Cecily O'Neill (Process Drama) unerwähnt. Weiterhin beschränkt sich die Auseinandersetzung mit Arbeiten von Schewe, Tselikas, Huber und Kessler auf Zusammenfassungen, die die Verfasserin in teilweise recht langen Fußnoten aus persönlicher Sicht („,meiner Meinung nach“) kommentiert; eine kritische wissenschaftliche Auseinandersetzung, in der Bezüge zwischen unterschiedlichen Ansätzen oder Vorgehensweisen hergestellt werden, ist das nicht.

Das zentrale Problem geht jedoch über die Aneinanderreihung unterschiedlicher Theorie- und Praxiskonzepte hinaus. Die Autorin selbst scheint performativen Ansätzen ambivalent gegenüberzustehen. An unterschiedlichen Stellen im Buch argumentiert sie gegen einen Absolutheitsanspruch von Dramapädagogik bzw. Dramagrammatik, den eigentlich niemand behauptet hat, z.B.:

Grundsätzlich stimme ich [...] zu, dass man sich um eine Anbindung [von Unterrichtsinhalten] an die Lebenswelt der Lernenden bemühen sollte. Ich glaube aber nicht, dass dies ausschließlich mit den Mitteln der Dramapädagogik möglich ist. (S. 61)

Nun muss man natürlich sagen, dass im traditionellen modernen kommunikativen Fremdsprachenunterricht [...] ebenfalls möglichst authentische Lernsituationen geschaffen werden, sodass größtmögliche Authentizität nicht unbedingt ein Privileg der Dramapädagogik ist. (S. 68)

So ein Kampf gegen Windmühlen gibt Rätsel auf; die Autorin scheint ihr Vorhaben, dramagrammatisch zu arbeiten, bereits im Vorfeld argumentativ zu sabotieren. Sie wendet sich explizit gegen den Einsatz von Fiktion und argumentiert, dass performative Handlungskontexte pseudo-authentisch sind und „konstruierte Realitäten“ (S. 63) darstellen, aus denen der Lernende „keine Rückmeldung zu seinem sprachlichen Handeln in der echten fremdsprachlichen Kommunikation" (S. 64) bekommt. 
Dieser Eindruck erhärtet sich angesichts der Modifikationen, die Schappert für ihre empirische Untersuchung vorgenommen hat. Um der Zielgruppe von Analphabet:innen gerecht zu werden, die auf wenig vorhergehende Lernerfahrungen zurückgreifen können, ersetzt Schappert dramagrammatische Aktivitäten wie kooperative Regelfindung in der Einordnungsphase und Diskussion in der Reflexionsphase durch zusätzliche grammatische Erklärungen, Beispiele und Übungen. Weiterhin werden Aktivitäten vermieden, die spontanes Sprechen im Rahmen fiktiver Realitäten erfordern; stattdessen agieren Teilnehmende in gelenkten und vorher geübten Dialogen als sie selbst.

An dieser Stelle soll nicht behauptet werden, dass diese Modifikationen falsch sind. Schappert hat auf Basis ihrer Erfahrungen und Überlegungen pragmatische Entscheidungen getroffen, die sie für eine Gruppe von Analphabet:innen am sinnvollsten erachtet, und begründet diese Entscheidungen auch. Nur dass das Endprodukt kaum noch von traditionellem kommunikativem Unterricht zu unterscheiden ist.

Aus diesem Grunde dürfte es dann auch kaum verwundern, dass die Datensätze der empirischen Studie wenig Aussagekräftiges ergeben. Manche Datensätze scheinen die Forschungshypothesen eher zu bestätigen, andere wiederum eher nicht. Es ist Schappert zu Gute zu halten, dass sie die Ergebnisse der empirischen Studie nicht wegargumentiert.

\begin{abstract}
Keine der beiden Methoden [Dramagrammatik vs. Kommunikative Methode, SE] ist folglich eindeutig besser oder schlechter, was in der Konsequenz für die dramapädagogischen Methoden (zumindest in Bezug auf den untersuchten Einsatz zur Grammatikvermittlung) bedeutet, dass mit ihnen der Kanon der zur Verfügung stehenden Methoden zur Vermittlung von Sprache um eine Methode erweitert werden kann. (S. 251)
\end{abstract}

Dieses Fazit lässt jedoch jüngere Fachdiskussionen unerwähnt, die über das Methodenkonzept hinausgehen und mit dem Begriff postmethod (Kumaravadivelu, 2009) neue konzeptuelle Wege in der performativen Fremdsprachendidaktik einschlagen haben (Even, 2011; Miladinović, 2019). Dabei gehen Schapperts Abschlussüberlegungen bzgl. Zeiterfordernis und Einsetzbarkeit dramagrammatischer Unterrichteinheiten, äußeren und inneren Lernbewegungen, Unterrichtsatmosphäre, Interaktionsformen und der Tatsache, dass manche Grammatikthemen sich für eine dramagrammatische Erarbeitung mehr anbieten als andere, durchaus in diese Richtung und sollten unbedingt weiterverfolgt werden.

\title{
Bibliografie
}

Even, S. (2003). Drama Grammatik. Dramapädagogische Ansätze für den Grammatikunterricht Deutsch als Fremdsprache. Iudicium. 
Even: Rezension von Mit Dramagrammatik zu grammatikalischer Kompetenz

Even, S. (2011). Drama grammar: Towards a performative postmethod pedagogy. Language Learning Journal, 39(3), 299-312. https://doi.org/10.1080/09571736.2010.543287

Kumaravadivelu, B. (2009). Understanding language teaching. From method to postmethod. Routledge.

Miladinović, D. (2019). Prinzipien eines performativen Fremdsprachenunterrichts. In S. Even, D. Miladinović \& B. Schmenk (Eds.), Lernbewegungen inszenieren. Performative Zugänge in der Sprach-, Literatur- und Kulturdidaktik. Festschrift für Manfred Schewe zum 65. Geburtstag (pp. 722). Narr Francke Attempto. 